\title{
IMAGING COLUMNS OF THE LIGHT ELEMENTS CARBON, NITROGEN AND OXYGEN WITH SUB ÅNGSTROM RESOLUTION
}

\author{
C. Kisielowski ${ }^{1}$, C.J.D. Hetherington ${ }^{1,2}$, Y.C. Wang ${ }^{1,3}$, R. Kilaas ${ }^{1}$, M.A. O'Keefe ${ }^{1}$, and \\ A. Thust ${ }^{4}$
}

1) National Center for Electron Microscopy, Material Sciences Division, Ernest Orlando Lawrence Berkeley National Laboratory,

One Cyclotron Road, Berkeley CA 94720, USA

2) University of Oxford

Department of Materials

Parks Road, Oxford OXI 3PH, UK

3) FEI Company, 7451 N.W. Evergreen Parkway, Hilsboro, OR 97124, USA

4) Institut fuer Festkoerperforschung, Forschungszentrum Juelich $\mathrm{GmbH}$, D-5242 Juelich, Germany

\begin{abstract}
It is reported that lattice imaging with a $300 \mathrm{kV}$ field emission microscope in combination with numerical reconstruction procedures can be used to reach an interpretable resolution of about $80 \mathrm{pm}$ for the first time. A retrieval of the electron exit wave from focal series allows for the resolution of single atomic columns of the light elements carbon, nitrogen, and oxygen at a projected nearest neighbor spacing down to $85 \mathrm{pm}$. Lens aberrations are corrected on-line during the experiment and by hardware such that resulting image distortions are below $80 \mathrm{pm}$. Consequently, the imaging can be aberration-free to this extent. The resolution enhancement results from increased electrical and mechanical stability's of the instrument coupled with a low spherical aberration coefficient of $0.595 \pm 0.005 \mathrm{~mm}$.
\end{abstract}

PACS: 07.80, 61.16.-d , 68.35.-p 


\section{DISCLAIMER}

This document was prepared as an account of work sponsored by the United States Government. While this document is believed to contain correct information, neither the United States Government nor any agency thereof, nor The Regents of the University of California, nor any of their employees, makes any warranty, express or implied, or assumes any legal responsibility for the accuracy, completeness, or usefulness of any information, apparatus, product, or process disclosed, or represents that its use would not infringe privately owned rights. Reference herein to any specific commercial product, process, or service by its trade name, trademark, manufacturer, or otherwise, does not necessarily constitute or imply its endorsement, recommendation, or favoring by the United States Government or any agency thereof, or The Regents of the University of California. The views and opinions of authors expressed herein do not necessarily state or reflect those of the United States Government or any agency thereof, or The Regents of the University of California. Ernest Orlando Lawrence Berkeley National Laboratory is an equal opportunity employer.

\section{Introduction}

In recent years scientists have developed the ability to create materials by design on an atomic level. It has become possible to formulate a structure atom-by-atom in order to allow it to perform a specific function ${ }^{1}$. This technology must be complemented by the development of novel instrumentation that is capable of analyzing such materials on a single atomic scale in order to compare theory with experiments. High Resolution Transmission Electron Microscopy [HRTEM] is the principle technique that can provide this ability. However, a typical point resolution around $0.2 \mathrm{~nm}^{2}$ is often insufficient to resolve mono atomic columns in many materials systems. Figure 1 depicts this situation by plotting the band gap energy of different ceramics and semiconductors versus their inter atomic spacing. It is seen that the resolution of single atomic columns in ceramiclike materials is barely possible. In such materials systems an additional complication arises from the low electron scattering power of light elements such as carbon, nitrogen or oxygen that are often located close to heavier elements. In practice, the separation of 
single atomic columns requires a point resolution that is much better than the $0.15 \mathrm{~nm}$ limit that is depicted in Figure 1. Lattice imaging along a zone axis of crystals nearly always shortens the inter atomic spacing by the projection into the image plane. For example, the resolution of dumbbell images along the commonly recorded [110] zone axis of silicon, cubic gallium nitride and diamond would require a point resolution of $0.136 \mathrm{~nm}, 0.113 \mathrm{~nm}$, and $0.089 \mathrm{~nm}$, respectively, as shown in Figure 2. However, HRTEM's with a point resolution of better than $0.16 \mathrm{~nm}$ are rare and the dumbbell structure of silicon is commonly not resolvable.

Resolution in electron microscopy was steadily improved in the last decade to reach 0.1 $\mathrm{nm}$ or less. Four major routes were pursued to achieve this goal. I: Incoherent scanning transmission electron microscopy [STEM] was utilized to demonstrate a point resolution of $0.078 \mathrm{~nm}^{3}$. II: Coherently scattered electrons in a high voltage instrument operating at 1.25 MV allowed for a point resolution between 0.095 and $0.089 \mathrm{~nm}{ }^{4,5}$. A $0.109 \mathrm{~nm}$ separation of silicon and carbon columns in $\mathrm{SiC}$ was resolved with a similar microscope ${ }^{6}$. Very recently, a calculated information limit of $\sim 70 \mathrm{pm}$ was reported for a $1 \mathrm{MV}$ HRTEM that is equipped with a cold field-emission electron source ${ }^{7}$. III: With the most commonly used instruments, i.e. those that operate in the mid-voltage range around 300 $\mathrm{kV}$ and use coherently scattered electrons, an information limit of $0.10 \mathrm{~nm}$ was reported already in $1993^{8}$. Electron holography exploited this information limit down to $0.104 \mathrm{~nm}$ ${ }^{9}$. Alternatively, it was reported that a direct reconstruction of the phase and the amplitude of the scattered electron wave from a focal series of HRTEM images is suitable to extend the interpretable resolution of a field emission microscope down to its information limit $^{10}$. Several approaches were published to tackle the problem of resolution extension $11,12,13,14,15,16$ with an earliest contribution suggested by Schiske ${ }^{17}$. Subsequently, phaseretrieval electron microscopy was developed into a generally applicable tool that extended the resolution of $200 \mathrm{kV}$ and $300 \mathrm{kV}$ field emission electron microscopes to values around $0.14 \mathrm{~nm}^{18,19,20,21}$ and provides the possibility to correct lens aberrations by software $^{22}$. A third variation of this theme is the tilt azimuth series in which four images are taken with the incident electron beam tilted to plus/minus $\mathrm{x}$ and $\mathrm{y}$ directions followed by reconstruction of the exit surface wavefunction ${ }^{23)}$. IV: Another successful approach to 
improve the point resolution consists of an active compensation of the spherical aberration of the objective lens by a hexapole corrector system ${ }^{24}$.

In this contribution we report that interpretable sub Ångstrom resolution can be achieved with a Philips CM300 field emission microscope. For the first time we report an interpretable resolution of better than $0.085 \mathrm{~nm}$ that is close to the $0.080 \mathrm{~nm}$ information limit that one expected from such an instrument ${ }^{25}$. A focal series reconstruction package $\mathrm{e}^{20}$ was employed to extend the point resolution of $0.168 \mathrm{~nm}$ of the instrument towards its information limit. The dominant 3-fold astigmatism was corrected by hardware to a residual value of $A_{2} \leq 50 \mathrm{~nm}$. This correction leads to image distortions that are smaller than $80 \mathrm{pm}$. Some of the results were published earlier ${ }^{26,27,28}$.

The paper is organized in the following manner. Section II summarizes experimental details of the sample preparation procedure and the image recording process. A brief description of the underlying physics together with results of performance tests is given in section III. In section IV we describe the implementation of the reconstruction procedure that includes tests on simulated lattice images of partial dislocation cores in silicon [110]. Experimental results are outlined in section $\mathrm{V}$ that is followed by a discussion (VI). Section VII summarizes and concludes the paper.

\section{Experimental details}

Thin films of gallium nitride and diamond were used for this investigation. The $2 \mu \mathrm{m}$ thin GaN crystals were deposited by Molecular Beam Epitaxy (MBE) on sapphire substrates $^{29}$. Similarly thick diamond films were grown by Chemical Vapor Deposition (CVD) on silicon ${ }^{30.31}$. Additionally, silicon test samples were utilized for calibration purposes. They contained a $1.3 \mathrm{~nm}$ thin amorphous tungsten layer sandwiched between two similarly thin layers of amorphous silicon. The structure was sputtered on Si [001]. A cross-section view is shown in Figure 3. The top layer oxidized to form $\mathrm{SiO}_{2}$ upon exposure to air. The tungsten layer is thin enough to allow for decent lattice imaging in a

plane-view configuration (Figure 3). This tungsten layer is used to tag the exit plane of the sample because its signal dominates the noise in the lattice image. 
The sample thickness commonly limits the achievable resolution at a given acceleration voltage because of a delocalization of the scattered electrons that increases with sample thickness $^{32}$. For the case of Si [110], Figure 4 shows that a resolution of the dumbbell structure in the phase of the electron exit wave can only be achieved with an acceleration voltage of $300 \mathrm{kV}$ in samples that are less than $10 \mathrm{~nm}$ thin. Improvements of the sample preparation procedure are necessary, because a typical ion-milling process creates samples with an undesirable large surface roughness. Holes that can be considerably deeper than $3 \mathrm{~nm}$ were revealed experimentally by measuring the surface topology with QUANTITEM $^{33,34}$ in a plan-view configuration. In addition, the required small sample thickness makes beam damage from the high brightness field emission gun a severe problem that needs to be slowed down to record a focal series of images. Finally, a 1-2 $\mathrm{nm}$ thin amorphous layer should be present on the sample surface because it is beneficial to record Thon rings ${ }^{35}$ that were used to measure focus settings.

In the course of this investigation, the requirements above were best met by mechanically thinning the samples to a thickness of a few microns and subsequently milling them with 2-3 kV argon ions in a Fishione or a Gatan ion mill. Argon ions that were accelerated by voltages as low as $200 \mathrm{~V}-500 \mathrm{~V}$ and hit the sample with a $3^{0}-5^{0}$ shallow incident angle ${ }^{36}$ were used as a final surface polish. Thereafter, the specimens were cleaned in an oxygen/argon plasma and a 1-2 nm thin amorphous carbon film was deposited on their backside. This layer serves three purposes: First, it slows down knock-on damage. Second, the amorphous layer provides an electrical path for discharging insulating samples such as sapphire. Finally, it generates the Thon rings close to the plane where the electrons exit the sample.

Figure 5 shows a lattice image of a silicon [110] sample. It is seen that the image pattern varies negligibly over a $20 \times 20 \mathrm{~nm}^{2}$ large field of view and that the $0.136 \mathrm{~nm}$ spacing of the dumbbells is visible throughout the image. From image simulations we find that the surface roughness should be less than $2 \mathrm{~nm}$ for this $6 \mathrm{~nm}$ thin sample. Similar results were reported for $4 \mathrm{~nm}$ thin aluminum $[100]^{37}$ that was prepared in an identical manner. More details of this sample preparation procedure will be published elsewhere ${ }^{38}$.

The Philips CM300 FEG/UT instrument with the described attachments is named One Ångstrom Microscope (OAM) at the NCEM. It meets specifications similar to those that 
were put forward within the Brite Euram Project No. $3322^{25}$. Lattice images were recorded through the attached GATAN Image Filter (GIF) that provides an additional magnification factor of 38 . The $24 \mu \mathrm{m}$ large pixels of the attached $2 \mathrm{k}$ X $2 \mathrm{k}$ CCD camera were binned by two to obtain an effective pixel size of $48 \mu \mathrm{m}$. A sampling rate of $10-20$ $\mathrm{pm} / \mathrm{pixel}$ is typical for our experiments and gives a magnification of $4.8-2.4 \times 10^{6}$. A spread of information across pixels ${ }^{39}$ gives rise to a magnification dependent resolution that was minimized by the binning process. In the best case we obtained $85 \mathrm{pm}$ of resolution recorded on 4.25 effective pixels with a magnification of $2.4 \times 10^{6}$.

Focal series up to 47 images were recorded with time intervals of 5 seconds between successive images with one second exposure time. We observed a typical image drift in the range of $80-110 \mathrm{pm}$ between successive lattice images that was determined by cross correlation from the experiments on sapphire, gallium nitrite and diamond that are reported here. In best cases, drift velocities as low as $10 \mathrm{pm} / \mathrm{second}$ were obtained. Therefore, the average image drift during exposure was $18-22 \mathrm{pm}$ and we used sampling rate of $20 \mathrm{pm} /$ pixel to assure that image drift does not limit the resolution.

Prior to each recording process the electron beam was aligned along the optical axis and the 2-fold astigmatism and the axial coma were corrected on-line. The 3-fold astigmatism was corrected previously by hardware ${ }^{27,40}$ to below $50 \mathrm{~nm}$ and is largely unaffected by the sample. Thereby, aberration free imaging down to $80 \mathrm{pm}$ of residual image distortions can be obtained. Principally, all residual aberrations can alternatively be corrected by software post aquisition ${ }^{22}$. However, in this work it was attempted to remove the mentioned aberrations already before recording and to work with as-recorded data.

Further details about the implementation of the OAM at the NCEM are published in Reference 40.

Image simulations were performed with NCEM's Image simulation software package NCEMSS ${ }^{41)}$.

III. Background and performance tests 
Isotropic information transfer of the OAM down to $0.10 \mathrm{~nm}$ was probed with Young's fringes of amorphous tungsten ${ }^{40}$. In this contribution, we investigated whether transmission of crystalline reflection in the sub Ångstrom region is possible.

In a coherent approximation and under exact focus conditions the information transfer is limited by spatial damping envelope

$$
E_{s}(\mathbf{u})=\exp \left[-(\pi \alpha / \lambda)^{2}\left(C_{s} \lambda^{3} u^{3}+f \lambda u\right)^{2}\right]
$$

to the Contrast Transfer Function (CTF) ${ }^{15,42}$.

$$
\sin \chi(u)=\sin \left(C_{s} \lambda^{3} u^{4} \pi / 2+\pi f \lambda u^{2}\right)
$$

Here, $\lambda$ is the electron wavelength, $u$ is a reciprocal lattice vector, $\alpha$ is the semi angle of the illuminating convergence cone, and $\mathrm{f}$ is the defocus.

However, a temporary damping envelope produced by mechanical and electrical instabilities imposes an information limit. This envelope is given by ${ }^{15,42}$ :

(3) $\mathrm{E}_{\mathrm{t}}(\mathbf{u})=\exp \left[-1 / 2 \pi^{2} \lambda^{2} \Delta^{2} \mathrm{u}^{4}\right]$

where $\Delta$ models the 1/e half width of a Gaussian spread of focus due to chromatic aberrations which can be approximated by ${ }^{15,25,42}$ :

(4) $\Delta=\mathrm{C}_{\mathrm{c}}\left[4(\Delta \mathrm{I} / \mathrm{I})^{2}+(\Delta \mathrm{E} / \mathrm{eV})^{2}+(\Delta \mathrm{V} / \mathrm{V})^{2}\right]^{1 / 2}$

$\mathrm{C}_{\mathrm{c}}$ is the coefficient of chromatic aberration. $\Delta \mathrm{E}$ is the thermal energy spread of the electrons of charge e and $\Delta \mathrm{I} / \mathrm{I}$ and $\Delta \mathrm{V} / \mathrm{V}$ are the relative fluctuations of lens current and high voltage, respectively.

Spatial frequencies that are recorded down to the Scherzer point resolution ${ }^{25}$

$$
\rho_{\mathrm{p}}=0.65 \mathrm{C}_{\mathrm{s}}^{1 / 4} \lambda^{3 / 4}
$$

exhibit the same sign. However, misphased information can be transferred far beyond this point resolution to a $1 / \mathrm{e}^{2}$ limit that is imposed by partial coherence that can be spatial ${ }^{25}$

$$
\rho_{\mathrm{s}}=\left(\pi \alpha \mathrm{C}_{\mathrm{s}} \lambda^{2}\right)^{1 / 3}
$$

or temporal ${ }^{40,25}$ :

$$
\rho_{\mathrm{t}}=\left(\pi \mathrm{C}_{\mathrm{c}} \lambda / 2\right)^{1 / 2}\left(4(\Delta \mathrm{I} / \mathrm{I})^{2}+(\Delta \mathrm{E} / \mathrm{eV})^{2}+(\Delta \mathrm{V} / \mathrm{V})^{2}\right)^{1 / 4}
$$

Reflections from diamond [110] are calculated together with the CTF of the OAM in Figure 6. It is seen that the diamond (004) reflection would not be transmitted around Scherzer defocus. However, it must appear in lattice images if the CTF is tuned to a larger underfocus if the temporal damping envelopes provide an information limit that is 
small enough. Typical imaging parameters that are used in this work are listed in Figure 6 , too.

Figure 7 depicts that the $0.091 \mathrm{~nm}$ spacing of the $\{222\}$ image Fourier coefficients from molybdenum [112] are directly visible in a lattice image. They cannot be excited by non linear beam interference's because they are of the lowest order as shown by the Fourier transform of the image. Similar images were obtained repeatedly to assure that information transfer at $91 \mathrm{pm}$ is routinely achievable.

In a second set of experiments, the defocus dependence of the (004) Fourier amplitude in diamond [110] was investigated. Dynamic calculations of the (002) and (004) beam amplitudes are shown in Figure 8a. The (004) beam amplitude is either similar or larger than the (002) one if the sample $8 \mathrm{~nm}$ thin or thinner. Contrary to these calculations it is seen from the experiment shown in Figure $8 b$ that the (004) Fourier amplitude is almost absent at an underfocus of $-108 \mathrm{~nm}$ even though the sample is only $6 \mathrm{~nm}$ thin. This is the effect of the spatial damping envelope. At an underfocus of $-207 \mathrm{~nm}$ the (004) Fourier amplitude exceeds the (002) Fourier amplitude as it is expected from the calculations. A measurement of the amplitude ratio $\mathrm{I}(400) / \mathrm{I}(200)$ from a focal series is shown in Figure $8 \mathrm{c}$ together with a calculation of the corresponding ratio of the spatial envelope calculated from equation (1) at frequencies $u_{400}$ and $u_{200}$. It is seen that in our experiment the (004) Fourier amplitude becomes significant around $\mathrm{f}=-170 \mathrm{~nm}$ which is the point where the edge of the of the spatial envelope sweeps across the spatial frequency $\mathrm{u}_{400}=($ $0.89 \mathrm{~nm})^{-1}$. The experiment implies that the information limit of the microscope lies beyond this value. The scatter of the experimental data for $\mathrm{f}<-170 \mathrm{~nm}$ is caused by the CTF oscillations which induces rapid oscillations of the Fourier amplitude. This oscillation or Fourier period is given by ${ }^{43}$ :

(8) $f_{p}=2 / \lambda u^{2}$

On the other hand, the limits given by equations (5) - (7) can be calculated because the typical root mean square $(\mathrm{RMS})$ values of the parameters $(\Delta \mathrm{I} / \mathrm{I})=0.5 \mathrm{ppm},(\Delta \mathrm{V} / \mathrm{V})=0.5$ ppm and $(\Delta \mathrm{E} / \mathrm{eV})=1.3 \mathrm{ppm}$ are $\mathrm{known}^{25}$ and $\mathrm{C}_{\mathrm{c}}$ was measured to be $(1.27 \pm 0.03) \mathrm{mm}$ utilizing equation (4). They are:

$\rho_{\mathrm{p}}=0.168 \mathrm{~nm}$

$\rho_{\mathrm{t}}=0.083 \mathrm{~nm}$ 
$\rho_{\mathrm{s}}=0.105 \mathrm{~nm}$

The spatial damping envelope $\rho_{\mathrm{s}}$ limits the achievable information transfer under Scherzer imaging conditions. However, it is well known that this value can be tuned to be comparable with $\rho_{t}$ if the objective lens is further underfocused and $C_{s}$ is sufficiently small $^{42)}$. This is demonstrated in the experiments on diamond [110] (Figure 8). Thus, the information limit from the temporal damping envelope exceeds the point resolution by a factor of two and reaches $0.08 \mathrm{~nm}$.

\section{Implementation of Exit Wave Reconstruction}

The restoration of properly phased scattered electron beams was solved within the Philips Brite Euram project No. $3322^{20,22}$. There, it was demonstrated that the electron exit wave of crystal structures and interfaces could be retrieved from a focal series of lattice images. A developed software package uses a parabolic - and a maximum likelihood algorithm that makes use of an equidistant focus settings of successive images to exploit parallel computation. No prior knowledge of the crystal structure is needed but instrumental parameters must be known to a sufficient accuracy.

At the NCEM the program was tested on simulated focal series from $90^{\circ}$ partial dislocation in silicon with different core structures ${ }^{28,44}$. A relaxed atomic structure around the dislocation cores was calculated using Tersoff potentials ${ }^{45}$. Figure 9 compares lattice images that one would obtain from NCEM's Atomic Resolution Microscope that operates at $1000 \mathrm{kV}$ and exhibits a point resolution of $0.16 \mathrm{~nm}$ with the retrieved phase of electron exit wave that one would obtain by reconstruction of a focal series from the OAM. Clearly, $0.16 \mathrm{~nm}$ of point resolution cannot separate the dumbbell structure of silicon [110] and would not allow to recognize a different core structure in the presence of experimental noise. Unlike the ARM, a focal series restoration with the OAM would fully recover the input structure with an interpretable resolution that largely exceeds $0.136 \mathrm{~nm}$.

A focal series restoration requires knowledge of the defocus setting for each image of a series. For this purpose an automated fitting procedure was developed that matches the CTF given by equation (2) to the Thon rings that are present in every recorded lattice image $^{46}$. A specific example is shown in Figure 10. The focus values of the experiments 
that are reported here are depicted in Figure 11. Reconstruction's were done with a minimum of 10 images and a maximum of 20. The plot reveals a linear dependence of the focus step size $\Delta \mathrm{f}$ with the recorded image number. This value is a crucial input parameter for the reconstruction software. A repeated recording of focal series from $\mathrm{Si}$ [001] reveals fluctuation of $\Delta \mathrm{f}$ that can be as large as $10 \%$ which is why we determine its value afresh for each series. Resultantly, a precision of better than $1 \%$ could be reached. The well-known lattice constant of silicon $(0.5429 \mathrm{~nm})$ was used to calibrate the sampling rate. Lattice parameters of $\mathrm{GaN}^{47}$ and sapphire ${ }^{48}$ were not suited for this purpose because they easily vary by $2 \%$ or more. The residual GIF distortions are typically $0.7 \%$ and can reach a minimum value of $0.25 \%$. They can be further reduced by measuring their effect on a mask of known geometry and then using these values to correct the recorded lattice images by software.

Figure 12a shows the Fourier periods of two orthogonal $\{220\}$ image Fourier coefficients from experiments on $\mathrm{Si}$ [001]. Suitable sine functions were fitted to the data. They reveal Fourier periods of $37.9 \pm 0.5 \mathrm{~nm}$ and $36.9 \pm 0.4 \mathrm{~nm}$, respectively which agree with the $37.3 \mathrm{~nm}$ value calculated from equation (8) within statistical errors. The two Fourier periods are asynchronous in defocus by $2 \mathrm{~nm}$ which is caused by a residual 2 -fold astigmatism. Thus, this procedure provides an accurate measurement of the 2-fold astigmatism provided it is small and the main axes of the elliptical distortions coincide with the directions of the two analyzed Fourier amplitudes. In the experiment this is true to within 10 degrees.

For the reconstruction process $\mathrm{C}_{\mathrm{S}}$ that must be known to within $10 \mu \mathrm{m}$ to prevent phase inversion for reflections from the sub Ångstrom region. Its value can be determined by measuring Fourier periods of Fourier amplitudes and Thon rings simultaneously because the spacing of the Thon rings depends on Cs (equation 2) while the Fourier period does not (equation 8). In the case of silicon [100] for example, the determination of the focal increment $\Delta \mathrm{f}$ between successive images from Thon rings and from Fourier periods of the [220] beams gives a starting value of $0.60 \mathrm{~mm}$ for the spherical aberration constant. It can be further refined by varying $\mathrm{C}_{\mathrm{s}}$ and $\mathrm{f}$ over a certain parameter range in the reconstruction procedure to obtain agreement between measured exit waves and simulations. The parameter range of interest was evaluated by a $\chi 2$ merit function 


$$
\chi 2\left(\mathrm{C}_{\mathrm{s}}, \mathrm{f}\right)=\Sigma_{\mathrm{i}=1}^{\mathrm{N}}\left[\left(\mathrm{y}_{\mathrm{i}}-\mathrm{f}_{\text {model }}\left(\mathrm{C}_{\mathrm{s}, \mathrm{i}}, \mathrm{f}_{\mathrm{i}}\right) / \sigma_{\mathrm{i}}\right]^{2}\right.
$$

where $y_{i}$ is the measured amplitude of the $\{220\}$ Fourier coefficients in image $i$ of a series, $\sigma_{\mathrm{i}}$ is its standard deviation, and $\mathrm{f}_{\text {model }}\left(\mathrm{C}_{\mathrm{s}, \mathrm{i}}, \mathrm{f}_{\mathrm{i}}\right)$ is the model function that relates $\mathrm{C}_{\mathrm{s}, \mathrm{i}}$ and $f_{i}$ which is given by use of equation (2). The resulting $\chi 2$ map from the experiment on $\mathrm{Si}$ [100] is shown in Figure 12b. There, dark regions mark good agreement between experiment and model. The defocus values refer to the first image of a series. If this defocus is extracted from Thon rings it leads to a small $\mathrm{C}_{\mathrm{s}}=0.52 \mathrm{~mm}$. The simulated exit wave from Si [100] matches badly the experiment in this case. Therefore, we repeated reconstructions of the electron exit wave with values $C_{s}$ and $f$ of minimal $\chi^{2}$ in order to obtain the best match of amplitude- and phase images between simulations and experiments. Details of the procedure will be given elsewhere ${ }^{49}$.

Figure 13 depicts the amplitude and the phase of the electron exit wave of Si [100] that was reconstructed from 20 lattice images and matches the simulation best. One of the lattice images is shown in Figure 3. The reconstruction is homogeneous over a $10 \times 10$ $\mathrm{nm}^{2}$ large field of view. The magnified image of Figure 14a compares the experiment with image simulations for a $12 \mathrm{~nm}$ thick sample. Simulated and experimental phase and amplitude images match well. They are separated in the exit wave reconstruction process. A further comparison of the normalized amplitudes of the simulation and the experiment is produced in Figure 14b. Quantitatively, a peak width of $70 \mathrm{pm}$ in the simulation compares with $80 \mathrm{pm}$ in the experiment. Usually, simulated and experimental phase images match well but the compared amplitude images may exhibit discrepancies. We argue that this difficulty is related to the smaller contrast of amplitude images in thin samples which are dominantly phase objects. It is for this reason that we exhibit phase images mostly.

Comparably good results for the experiments with Si [100] were obtained for $0.58 \mathrm{~mm} \leq$ $\mathrm{C}_{\mathrm{s}} \leq 0.60 \mathrm{~mm}$ and corresponding focus values around $-204 \mathrm{~nm}$ that were related to $\mathrm{C}_{\mathrm{s}}$ by Figure 12b. Similarly, a reconstruction of the exit wave of diamond [110] and sapphire [11-20] yield good results for $C_{s}=0.590 \pm 0.005$ and $C_{s}=0.600 \pm 0.005$, respectively. Therefore, we find that the spherical aberration constant of the OAM is $0.595 \pm 0.005$ $\mathrm{mm}$ which is independent of the samples position in the pole piece, only, if it is located in the same (eucentric) position. 
V. Detection of light elements with sub Ångstrom resolution

In this Section we demonstrate our ability to achieve sub Ångstrom resolution and detect the light elements $\mathrm{C}, \mathrm{N}$, and $\mathrm{O}$.

Figure 15a shows an experimental reconstruction of the phase of the electron exit wave from diamond [110]. The dumbbell structure of the carbon columns separated by $89 \mathrm{pm}$ is readily resolved for the first time. From images simulations we find that the sample is about $6 \mathrm{~nm}$ thin and that the experiment suffers from a substantial sample tilt of 10-15 mrad. A resolution of the dumbbell structure requires transmission of the $\{400\}$ reflections. Their presence in the reconstruction can be tested by the application of an aperture at $0.1 \mathrm{~nm}$ as shown in Figure 15b. It is seen that the $\{400\}$ reflections contribute substantially to the image formation because the dumbbell structure is smeared out in the filtered image.

In Figure 16 we show the reconstructed the exit wave of an interface between cubic (3C) and hexagonal $(2 \mathrm{H})$ gallium nitride. It is seen that nitrogen columns can be resolved for the first time. They are separated by only $113 \mathrm{pm}$ from the heavier gallium columns. To date, the large electron scattering by heavy elements made the detection of closely spaced light elements impossible if $\mathrm{z}$-contrast imaging is employed. Image simulations reveal that the sample is $10 \mathrm{~nm}$ thin and tilted by roughly $12 \mathrm{mrad}$. The location of the Ga and the $\mathrm{N}$ columns is determined by the lower contrast of the nitrogen columns. Delocalization effects from the field emission source were removed by the reconstruction. The structure of the interface is intuitively clear because the hexagonal a,c,a,c,a,c... stacking of basal planes in the hexagonal matrix must transforms into the cubic a,b,c,a,b,c... stacking of (111) planes as the interface is crossed. This is confirmed by the experiment.

Finaly, Figure 17a shows the reconstructed phase image of a GaN/sapphire interface that could not be investigated in the past with truly atomic resolution because of lacking resolution and sensitivity. At the interface, all atomic columns are resolved. A periodic array of misfit dislocations stands off the interface and extends into the GaN by a few 
atomic layers. A detailed analyses of the interface structure will be given elsewhere ${ }^{38}$. Here, we show in Figure $17 \mathrm{~b}$ a magnified view of the electron exit wave from the sapphire. A simulation of the exit wave is inserted into the picture which assumes an information limit of $80 \mathrm{pm}$. From the simulation we find that the crystal is $6 \mathrm{~nm}$ thin and tilted by $3 \mathrm{mrad}$ from the zone axis orientation with respect to the electron beam. It is seen that all oxygen and aluminum columns are resolved at a smallest separation of 85 pm. These Al-O columns are arranged in rows that extend diagonally across the image. The largest distortion in the image stems from the $3 \mathrm{mrad}$ of sample tilt that is large enough to cause a significant alteration of the intensity distribution in the lower one of the two parallel Al-O rows. It is remarkable that the interpretable resolution in the experiment compares well or even exceeds the one of the simulation. Consequently, the information limit of the microscope is around $80 \mathrm{pm}$.

\section{Discussion}

This experimental investigation aimed to extend the point resolution of a $300 \mathrm{kV}$ field emission microscope to values around one Ångstrom and possibly below. Focal series reconstruction $^{20}$, electron holography ${ }^{8}$ and extended resolution electron microscopy ${ }^{15}$ were pursued to achieve the goal. All three methods require computer processing and support to extract the desired structural information from the recorded lattice images or holograms. Out of the tested approaches, the reconstruction of the electron exit wave provided the best interpretable resolution of $80 \mathrm{pm}$ or even less. Electron Holography allowed us to transmit the diamond (222) reflection at $0.103 \mathrm{~nm}$ in the side bands at best. The extended resolution microscopy is currently being further developed. Therefore, we concentrate on a discussion of the exit wave reconstruction method and related experimental aspects.

The preparation of suitable samples becomes even more crucial if sub Angstrom resolution is attempted. Limiting factors are the local spread of information that increases with sample thickness and the roughening of the sample surfaces that is induced by the sample preparation. Figure 4 shows for the specific case of the dumbbell images in 
silicon [110] that a sample thickness of less than $10 \mathrm{~nm}$ is required for a resolution around $0.1 \mathrm{~nm}$. We tested currently available technologies on silicon samples and compared the results of a particular thinning process. Out of the tested instruments, the acquired low voltage and low angle ion mill process yield the best result in terms of surface smoothness. In the case of Si [110] (Figure 5) we found that thickness fluctuation may be substantially less than $2 \mathrm{~nm}$, which was a limit that was imposed by a comparison of experiments with simulations. More systematic studies are certainly desirable and improvements in sample preparation techniques will remain an issue.

We utilized materials of known structure to evaluate the interpretable resolution. The [110] projection of the materials $\mathrm{Si}$, cubic $\mathrm{GaN}$, and diamond provides similar - or identical lattice images and exit waves. It is convenient for test purposes that their inter atomic spacing decreases from $136 \mathrm{pm}$ in case of the silicon to a $89 \mathrm{pm}$ in diamond with its extreme short lattice parameter. With the resolution of single atomic carbon columns in the [110] orientation it is also demonstrated that it is now even possible to resolve the diamond lattice along three different zone axes namely along [111], [100], and [110].

In these materials the chemical species nitrogen and carbon are present. Sapphire [11-20] was investigated to test if single oxygen columns can be imaged at a projected nearest neighbor spacing as short as $85 \mathrm{pm}$. In the past, the resolution of the light elements carbon, nitrogen, oxygen with an atomic number $\mathrm{z}=6,7,8$ has been challenging. Experiments with inelastic electron scattering presently lack of sensitivity to detect $\mathrm{C}, \mathrm{N}$, or $\mathrm{O}$. Sensitivity is gained if $\mathrm{z}$ increases ${ }^{50}$. If the light element's next neighbors are significantly heavier, it is particularly difficult to detect them. This situation is found in $\mathrm{GaN}\left(\mathrm{z}_{\mathrm{Ga}}=31\right)$. Therefore, reports on the resolution of light elements are rare ${ }^{6,21}$ and restricted to experiments with elastically scattered electrons in materials where the projected nearest neighbor spacing is above one Ångstrom. It is for the first time that the dumbbell structure of diamond [110] is resolved, that single nitrogen columns are detected, and that oxygen columns in sapphire [1120] are resolved.

The reliability of the exit wave reconstruction procedure was tested and discussed in detail $^{20,22}$. We confirm that a reconstruction of simulated images is robust by recovering amplitude and phase from the simulated focus series of partial dislocations in silicon (Figure 9). At present, most problems arise on the experimental side. The benefits of 
reconstructing the electron exit wave are drastically visualized in Figure 9 where it is seen how much structural details can be gained by increasing the interpretable resolution from values around $0.16 \mathrm{~nm}$ for the ARM to values around $0.08 \mathrm{~nm}$ for the OAM.

Aberration free imaging was assumed in the simulations of Figure 9. Experimentally, we repeated measurements of the 3-fold astigmatism to assure that its hardware correction was stable and $50 \mathrm{~nm}$ (or less) over a time period of one year. Residual aberrations in the lattice images that arise from an imperfect on-line correction of the 2-fold astigmatism, the beam tilt and the coma can be small. In case of Si [100] we measured $2 \mathrm{~nm}$ of residual 2 -fold astigmatism. Thus, aberration free imaging down to the information limit is feasible. If necessary, any remaining distortion can be corrected by software ${ }^{22}$.

There are two ways to extract the focus value of all lattice images from a series. The first one consists of the analyses of Thon rings ( Figures $10 \& 11$ ) and the second approach utilizes the Fourier periods (Figure 12). A combination of the procedures allows to measure Cs. It is our experience that in both cases the extracted focus values do not coincide with the exit plane of the electron wave function. Usually the wave function must be propagated to its proper exit plane that we currently determine by a match of the experiments with simulations that can be achieved trough a minimization of the amplitude contrast. Typical propagation distances are comparable with the sample thickness and it is likely that these differences arise from uncertainties in the location of amorphous layers that coat the sample.

An analysis of the Fourier periods in combination with image simulations was employed to refine the $\mathrm{C}_{\mathrm{s}}$ measurement of our microscope. The procedure relates a change of focus with the necessary change of $\mathrm{C}_{\mathrm{s}}$ for a measured set of low indexed Fourier amplitudes. This method is currently being developed into a software package ${ }^{49}$. Our spherical aberration constant of $0.595 \pm 0.005 \mathrm{~mm}$ is small if the sample is located at the euzentric height and objective lens currents is kept constant. A value of $0.62 \pm 0.01 \mathrm{~mm}$ was reported before ${ }^{25}$.

The OAM has a nominal point resolution of $\rho_{p}=0.168 \mathrm{~nm}$. Close to Scherzer defocus, it is the spatial damping envelope $\rho_{\mathrm{s}}=0.105 \mathrm{~nm}$ that limits information transfer to this nominal value. Young fringes were recorded under these conditions and they extend to $0.10 \mathrm{~nm}^{40}$. Our experiments on molybdenum [112] prove sub Ångstrom information 
transfer (Figure 7). In addition, it is well known that the spatial damping envelope can be tuned to sub Ångstrom values if a larger underfocus is employed ${ }^{42}$. We utilized this effect to demonstrate sub Ångstrom information transfer in focal series from diamond [110] that extends towards Lichte defocus ${ }^{51}$. These experiments ensure that information at $89 \mathrm{pm}$ is passed by the objective lens at an underfocus of around -170 nm (Figure 8). Moreover, the amount as to which information is transferred in the sub Angstrom region was tested directly by the application of a $0.1 \mathrm{~nm}$ large aperture to the reconstructed phase image from the diamond [110]. The aperture wipes out the dumbbell structure to produce one elongated "blob". Thereby, we prove that the $\{400\}$ Fourier amplitudes are essential to the resolution of the diamond dumbbells (Figure 15b). Finally, a direct comparison of simulations and the experimentally reconstructed from sapphire [1120] (Figure 17b) shows that the information limit of the OAM is $80 \mathrm{pm}$ or even better.

Table I compares the results that were achieved in this study with literature data $^{3,4,5,7,9,10,20,23}$. The interpretable resolution was steadily improved to reach values close to one Ångstrom and beyond. The availability of field emission technology is a key element of this development. Its exploitation requires utilizing computational phase retrieval methods. By now, mid voltage microscopes have surpass the point resolution of high voltage microscopes that are equipped with thermal emitters. It is advantageous that their lower acceleration voltage causes less knock-on damage. High voltage FEG instruments could principally exhibit a better interpretable resolution but this has not yet been demonstrated. Our resolution compares well with recently published data from zcontrast measurements. Both phase retrieval microscopy as well as z-contrast microscopy produce images of electrons that are trapped in the potential of atomic columns which is sometimes referred to as "a picture of a 1 s state" ${ }^{52,53)}$. Our demonstrated capability to detect light elements together with a precision as to which atomic columns can be located in phase contrast microscopy that approaches $1 \mathrm{pm}^{21)}$ add unique advantages to the use of elastically scattered electrons.

Currently, the proper alignment of the sample along the electron beam remains challenging. The mechanical sample tilt installation allows for a smallest adjustment of $0.05^{\circ}$ ( $0.9 \mathrm{mrad}$ ) and it exhibits a hysteresis. As a result, a residual sample tilt of $1 \mathrm{mrad}$ and $3 \mathrm{mrad}$ was present in the experiments with silicon and sapphire, respectively. In case 
of the sapphire, the simulation in figure 15 shows that $3 \mathrm{mrad}$ of sample tilt causes a substantial intensity re-distributions in the phase image that can be already recognized by visual inspection. This puts limits on the precision as to which atomic positions can be extracted quantitatively.

Finally, we remark that the use of a 2 kx 2k CCD camera is essential for sub Angstrom experiments. First, this CCD provides an extra magnification factor of $\sim 40$ that allows for a magnification of $3 \times 10^{6}$ and more while the maximum magnification of $0.84 \times 10^{6}$ of the CM300 is insufficient. In addition, our experiments measuring its Modulation Transfer Function (MTF) agree with recently published data ${ }^{54)}$ that also established an improved performance of the $2 \mathrm{k} x 2 \mathrm{k}$ CCD compared with a $1 \mathrm{k} \times 1 \mathrm{k} C \mathrm{CD}$ if the $2 \mathrm{k} \mathrm{x} 2 \mathrm{k}$ CCD is operated with $2 \times 2$ pixel binning ${ }^{54)}$.

\section{Summary and Conclusion}

This contribution describes performance improvements of mid voltage microscopes that were steadily achieved in the Brit Euram Project No 3322 and at the NCEM over the last decade. Here, it is demonstrated for the first time that an information limit of $0.080 \mathrm{~nm}$ could be reached experimentally. The reasons for the achieved improvements are seen in a reduction of mechanical and electrical instabilities coupled with a small value of $\mathrm{C}_{\mathrm{s}}=$ $0.595 \pm 0.005 \mathrm{~mm}$. In addition, an on-line and hardware correction of the lens aberrations can be performed to an accuracy that allows for a direct interpretation of the reconstructed sub Ångstrom phase images. Overall, it is the goal of this investigation to make this tools available for materials sciences routinely.

The retrieval of exit waves from focal series can be reliably obtained within hours if the samples are suitable and a resolution close to one Ångstrom is desired. It is more time consuming to achieve sub Ångstrom performance. At present, sample preparation and sample tilt are severe bottlenecks for a routine achievement of sub Ångstrom results. Current research efforts at the NCEM focus on a quantification of atomic positions and the local chemistry.

It is remarkable that the use of elastically scattered electrons made it possible for the first time to produce images of the light elements carbon, nitrogen and oxygen with a 
projected next neighbor spacing close to one Ångstrom and below. This achievement allows to visit and to re-visit unsolved problems of materials sciences that involve interfaces, dislocations and nano structures. One expects the largest impact of electron microscopy with ultra high resolution and sensitivity for the detection of light elements in such fields of sciences.

\section{Acknowledgements}

The authors thank C. Nelson for supporting the day to day operation and maintenance of the OAM. C. Song, M. Kelsch, S. Sprater, and E. Stack helped extraordinarily to improve sample preparation techniques. Diamond thin film growth at Brown University is supported by the National Science Foundation under Award No. DMR-9619520 and was performed by B.W. Sheldon and A. Rajamani. A. Schwartzman suggested TEM investigations on this material. J. Wilson grew diamond films at the Heriot-Watt University that were investigated by A. Fitzgerald at the NCEM. J.-P Penisson provided the molybdenum samples. Y. Kim and E.R. Weber deposited the GaN thin films on sapphire at the University of Berkeley. J. Plitzko implemented the automatic acquisition of focal series. C. Kisielowski is grateful to D. van Dyck for commenting the manuscript. The project found continuously support through NCEM's head, U.Dahmen and was sponsored by the Director, Office of Science, Office of Basic Energy Sciences, of the U.S. Department of Energy under Contract No. DE-AC03-76SF00098.

\section{References:}

1) Workshop on Complex System, Ernest Orlando Lawrence Berkeley National Laboratory, PUB-826, 1999

2) M.A. O'Keefe, Ultramicroscopy 47 (1992) 282

3) P.D. Nellist, S.J. Pennycook, Phys. Rev. Letters 81 (1998) 4156

4) G. Moebus et al. J. Electron Microscopy 46 (1997) 381 
5) F. Phillipp in Proc. of the Asian Science Seminar on New Directions inTransmission Electron Microscopyand Nano-Characterization of Materials, eds. C.Kinoshita, Y. Tomokiyo, and S. Matsumura (Kyushu University Press, Fukuoka, ISBN4-87378558-8) (1998) 75

6) H. Ichinose, International Workshop on High Voltage and High Resolution Electron Microscopy, Stuttgard 1994, unpublished

7) T. Kawasaki, T. Yoshida, T. Madsuda, N. Osakabe, A. Tonomura, I. Matsui, K. Kitazawa, Appl. Phys. Lett. 76 (2000) 1342

8) H. Lichte, P. Kessler, F. Lenz, W.-D. Rau, Ultramicroscopy 52 (1993) 575

9) A. Orchowski, W.D. Rau, H. Lichte, Phys. Rev. Lett. 74 (1995) 399

10) W. Coene, G. Janssen, M. Op de Beeck, D. Van Dyck, Phys Rev. Lett. 69 (1992) 3743

11) W.O. Saxton, J. Microsc. Spectrosc. Electron. 5 (1980) 661

12) E.J. Kirkland, Ultramicroscopy 15 (1984) 151

13) D. Van Dyck, M. Op de Beeck, Proc. $12^{\text {th }}$ International Congress for Electron Microscopy, Seattle, Washington 1990, 26

14) W. Coene, $50^{\text {th }}$ Ann. Proc. EMSA, Boston, Massachusetts 1992, 986

15) M.A. O’Keefe in “Microstructure of Materials”, K. Krishnan ed. ; San Francisco Press Inc. 1993, 121

16) W.Z. He, F.H. Li, H. Chen, K. Kawasaki, T. Orkawa, Ultramicroscopy 70 (1997) 1

17) P. Schiske, in Proceedings $4^{\text {th }}$ European Conference on electron Microscopy, Rome 1968,145

18) W.M.J. Coene, A. Thust, M. Op de Beeck, D. Van Dyck, Ultramicroscopy 64 (1996) 109

19) M. Op de Beeck, D. Van Dycke, W. Coene, Ultramicroscopy 64 (1996) 167

20) A. Thust, W.M.J. Coene, M. Op de Beeck, D. Van Dyck, Ultramicroscopy 64 (1996) 211

21) C.L. Jia, A. Thust, Phys. Rev. Lett. 82 (1999) 5052

22) A. Thust, M.H.F. Overwijk, W.M.J. Coene, M. Lentzen, Ultramicroscopy 64 (1996) 249 
23) A. Kirkland, W. Saxton, K.-L. Chau, K. Tsuno, M. Kawasaki, Ultramicroscopy 57 (1995) 355

24) M. Haider, H. Rose, S. Uhlemann, E. Schwan, B. Kabius, K. Urban, Ultramicroscopy 75 (1998) 53

25) D. Van Dyck, H. Lichte, K.D. van der Mast, Ultramicroscopy 64 (1996) 1

26) J. H. Turner, M. A. O'Keefe and R. Mueller, in 55th Ann. Proc. MSA, Cleveland, Ohio 1997,1177

27) Y.C. Wang, A. Fitzgerald, E.C. Nelson, C. Song, M.A. O'Keefe and C. Kisielowski, in 57th Ann. Proc. MSA, Portland, Oregon 1999, 822

28) C. Kisielowski, A. Schwartzmann, C.E. Nelson, C. Song, R. Kilaas, A. Thust, Microscopy and Microanalysis 6 (2000) 14

29) M.S.H. Leung, R. Klockenbrink, C. Kisielowski, H. Fujii, J. Krueger, Sudhir, G.S., A. Anders, Z. Liliental-Weber, M. Rubin, E.R. Weber, Mat. Res. Soc. Proc. 449 (1997) 221

30) M.G.Jubber, J.I.B. Wilson, I.C. Drummond, P. John, D.K. Milne, Vacuum 45 (1994) 499

31) S. Nijhawan, S.M. Jankovsky, B.W. Sheldon, and B.L. Walden, J. Mater. Res. 14 (1999) 1046

32) M.A. O’Keefe, Microscopy \& Microanalysis 3 (1997) 1165

33) P. Schwander, C. Kisielowski, M. Seibt, F.H. Baumann, Y. Kim, A. Ourmazd, Phys. Rev. Letters 71 (1993) 4150

34) C. Kisielowski, P. Schwander, F.H. Baumann, M. Seibt, Y. Kim, A. Ourmazd, Ultramicroscopy 58 (1995) 131

35) F. Thon, Z. Naturforschung A20 (1965) 154

36) A. Brana, B. Pecz, M. Menyhard, Ultramicroscopy 70 (1998) 16

37) Plitzko, J.M., Campbell, G.H., King, W.E., Foiles, S.M., Advances in Materials Problem Solving with the Electron Microscope, MRS Symposium Boston, Massachusets 1999

38) C. Kisielowski, unpublished

39) R.R, Meyer, A. Kirkland, Ultramicroscopy 75 (1998) 23 
40) M.A. O’Keefe, C.J.D. Hetherington, Y.C. Wang, E.C. Nelson, J.H. Turner, C.

Kisielowski, J.-O. Malm, R. Mueller, J. Rignalda, M. Pan, A. Thust, Ultramicroscopy, this volume.

41) R. Kilaas, $45^{\text {th }}$ Ann. Proc. EMSA 1987, 66

42) D.B. Wiliams, C.B. Carter in "Transmission Electron Microscopy", Plenum Press, New York 1996

43) S. Jijima, M.A. O’Keefe, J. Microsc. 117 (1979) 347

44) The investigations were performed in a collaboration with M. Mills, M. Savage and D. Chrzan

45) J. Tersoff Phys. Rev. B 38 (1988) 9902

46) The program package can be downloaded from the NCEM web page at ncem.lbl.gov

47) C. Kisielowski in "Semiconductors and Semimetals" 57, J.I. Pankove and T.D. Moustakas eds., Academic Press, New York 1999, 257

48) P. Villars, L.D. Calvert in "Pearson's Handbook of Crystallographic Data", Vol.1, ASM International, Materials Park 1991, 970

49) R. Kilaas, C. Kisielowski, to be published

50) S.J. Pennycook, D.E. Jessen, Ultramicroscopy 37 (1991) 14

51) H. Lichte, Ultramicroscopy 38 (1991) 13

52) D. van Dyck, J.H. Chen, Solid State Communications 109 (1999) 501

53) S.J. Pennycook, B. Rafferty, P.D. Nellist, Microscopy and Microanalysis 6 (2000) 106

54) R.R. Meyer, A.I. Kirkland, R.E. Dunin-Borkowski, J.L. Hutchison, Ultramicroscopy 85 (2000) 9

Table 1:

Summary of a reported best resolution in experiments that use electron beams. 
Figure captions:

Figure 1:

Band gap energy versus inter atomic nearest neighbor spacing of common semiconductors and ceramics.

Figure 2:

Experimental [110] lattice images of $\mathrm{Si}, \mathrm{GaN}$, and Diamond. In this common projection a resolution of single atomic columns requires the recording of $\{004\}$ Fourier components at $136 \mathrm{pm}, 113 \mathrm{pm}$ and $89 \mathrm{pm}$, respectively. However, in single lattice images lattice images (004) Fourier components can originate from non-linear beam interferenes which do not contribute to resolution.

Figure 3:

Cross-section (left) and plane-view (right) lattice images of a silicon [100] test wafer that was capped with an amorphous tungsten layer.

Figure 4:

Simulated dumbbell images from silicon [110]. The phase of the electron exit wave was evaluated. Intensities were measured at the indicated positions $I_{a}, I_{b}$ and converted into contrast. Insets depict the broadening of the phase image with sample thickness that is caused by an increasing delocalization. For comparison with the phase images a simulated single lattice image is shown, too.

Figure 5:

A Si [110] sample prepared by low voltage $(500 \mathrm{~V}) /$ low angle $\left(3^{0}\right)$ ion milling. Left: Experimental lattice image. Right: magnified partial view. (004) Fourier components are resolved homogeneously over a large field of view giving rise to the "dumbbell" structure. The sample is $\sim 6 \mathrm{~nm}$ thin and recorded at a underfocus of $\sim-90 \mathrm{~nm}$. The homogeneous pattern reflects a smooth surface topology with possible thickness fluctuations of $2 \mathrm{~nm}$ and little local buckling ( $\leq 1 \mathrm{mrad})$. 
Figure 6:

Simulated contrast transfer functions (CTF) of the One Angstrom Microscope (OAM) for two commonly used focus settings. The point resolution $\left(\rho_{p}\right)$ and the information limit $\left(\rho_{\mathrm{s}, \mathrm{t}}\right)$ as well as reflections from diamond [110] are indicated. Instrumental parameters for the simulation are listed. Similar parameters are used for exit wave reconstructions.

Figure 7:

Experimental lattice image from molybdenum [112] (left) and its corresponding Fourier transform (right). The (222) Fourier coefficients at 91 pm cannot be excited non linearly and are readily visible in the lattice image.

Figure 8:

a) Calculated beam amplitudes of the (002) and the (004) beams in diamond [110] for a sample thickness below $8 \mathrm{~nm}$.

b) Experimental diamond [110] lattice images and their Fourier transforms for two different defocus settings of $-108 \mathrm{~nm}$ (left) and $-207 \mathrm{~nm}$ (right). The (004) Fourier component is visible at $\mathrm{f}=-207 \mathrm{~nm}$.

c) Experimental Amplitude ratio $\mathrm{I}(004) / \mathrm{I}(002)$ versus defocus. A corresponding envelope ratio was calculated from equation (1). The (004) reflection is attenuated by spatial envelope at focus settings above $\sim-170 \mathrm{~nm}$ and becomes dominant below this value.

Figure 9:

Image simulations of computed $90^{\circ}$ partial dislocation in silicon with two different core structures. The top row depicts lattice images that would be obtained from the Atomic Resolution Microscope (ARM) operating at $1 \mathrm{MV}$. The bottom row shows retrieved phase images of the electron exit wave that were obtained from 20 simulated lattice images if recorded by the OAM. 
Figure 10:

Defocus determination by fitting the CTF (equation 2) to the Thon rings of an experimental lattice image.

\section{Figure 11:}

Underfocus values determined from Thon rings versus experimental lattice image number of the considered focus series of silicon, sapphire, diamond, and $\mathrm{GaN}$

Figure 12:

Determination of Fourier periods, focus settings, and $\mathrm{C}_{\mathrm{s}}$ from silicon [001]:

a) Amplitude oscillations of two orthogonal $\{220\}$ Fourier components versus underfocus measured from Thon rings. Suitable sine functions are fitted to the experimental data. One oscillation period is half a Fourier period.

b) $\chi 2$ map for a range of test values $0.5 \mathrm{~mm} \leq \mathrm{C}_{\mathrm{s}} \leq 0.7 \mathrm{~mm}$ and $-185 \mathrm{~nm} \leq \mathrm{f} \leq-205 \mathrm{~nm}$. Small $\chi 2$ values reflect good agreement between the model function and the data. The defocus value from the Thon rings of the first lattice image is indicated and compared with the best value obtained from the exit wave reconstruction program.

Figure 13:

Experimentally recovered amplitude (left) and phase (right) of the reconstructed electron exit wave of silicon [100].

Figure 14:

a) Left: Magnified view of the amplitude and the phase of the reconstructed electron exit wave of silicon [100]. Image simulations (inset) are compared with the experiment .

b) Right: Comparison of amplitude oscillations in an experiment and a simulation. Line traces are taken in [001] direction. Amplitudes are normalized and are smaller in the experiment. A half width of $70 \mathrm{pm}$ in the simulation compares with $80 \mathrm{pm}$ in the experiment. A sample thickness of $\sim 12 \mathrm{~nm}$ causes the width of the peaks. 
Figure 15:

a)

Experimental phase image and inserted simulation of the electron exit wave from diamond [110]. Atomic positions are marked. Single carbon columns are resolved at a spacing of $89 \mathrm{pm}$.

b)

Top, left: Magnified phase image of the electron exit wave of diamond [110] as it is reconstructed.

Top right: Phase image after application of an aperture at $0.1 \mathrm{~nm}$ that allows for a transmission of $\{311\}$ and $\{222\}$ Fourier components at $0.107 \mathrm{~nm}$ and $0.103 \mathrm{~nm}$ but suppressed the $\{004\}$ Fourier components at $0.089 \mathrm{~nm}$. $\{004\}$ Fourier components cause the resolution of the dumbbell structure.

Bottom: Line traces across both structures. Their location is indicated in the phase images.

Figure 16:

Experimental phase image of the electron exit wave of an interface between cubic (3C) and hexagonal $(2 \mathrm{H}) \mathrm{GaN}$. Single nitrogen columns $113 \mathrm{pm}$ close to the gallium columns are resolved. The a,c,a,c...stacking sequence changes to the cubic a,b,c, a,b,c,.. stacking as the interface is crossed.

Figure 17:

a)

Experimentally reconstructed phase of the electron exit wave of the interface between $\mathrm{GaN}$ and sapphire. The interpretable resolution in the image is around $80 \mathrm{pm}$.

b) 
Magnified view of the electron exit wave from sapphire [1120]. A simulated phase image is inserted. Atom positions and chemical species are marked. Single oxygen columns are resolved that are separated by $85 \mathrm{pm}$ from the $\mathrm{Al}$ columns. The sample is $6 \mathrm{~nm}$ thin. 3 mrad of sample tilt is included in the simulation. The tilt is sufficient to cause substantial intensity shifts in the Al-O pairs which is why they do not appear identical in the parallel rows. 